\title{
A hard $x$-ray split-and-delay unit for the HED experiment at the European XFEL
}

\author{
Sebastian Roling ${ }^{1 *}$, Karen Appel ${ }^{2}$, Stefan Braun ${ }^{3}$, Alexey Buzmakov ${ }^{4}$, O. Chubar ${ }^{5}$, Peter Gawlitza ${ }^{3}$, \\ Liubov Samoylova ${ }^{2}$, Björn Siemer ${ }^{1}$, Evgeny Schneidmiller ${ }^{6}$, \\ Harald Sinn ${ }^{2}$, Frank Siewert ${ }^{7}$, Thomas Tschentscher ${ }^{2}$, Frank Wahlert ${ }^{1}$, Michael Wöstmann ${ }^{1}$, Mikhail \\ Yurkov $^{6}$ and Helmut Zacharias ${ }^{1^{*}}$ \\ ${ }^{1}$ Physikalisches Institut, WWU Münster, Wilhelm-Klemm Straße 10, 48149 Münster, Germany \\ ${ }^{2}$ European XFEL GmbH, Albert-Einstein-Ring 19, 22761 Hamburg, Germany \\ ${ }^{3}$ Fraunhofer IWS Dresden, Winterbergstraße 28, 01277 Dresden, Germany \\ ${ }^{4}$ Shubikov Institute of Crystallography RAS, 119333 Moscow, Russia \\ ${ }^{5}$ Brookhaven National Laboratory, Upton, NY 11973, USA \\ ${ }^{6}$ Deutsches Elektronen-Synchrotron, 22603 Hamburg, Germany \\ ${ }^{7}$ Helmholtz-Zentrum Berlin, Albert-Einstein-Straße 15,12489 Berlin, Germany \\ *corresponding authors: s_roli02@uni-muenster.de, h.zacharias@uni-muenster.de
}

\begin{abstract}
For the High Energy Density (HED) experiment [1] at the European XFEL [2] an x-ray split- and delay-unit (SDU) is built covering photon energies from $5 \mathrm{keV}$ up to $20 \mathrm{keV}$ [3]. This SDU will enable time-resolved $\mathrm{x}$-ray pump / $\mathrm{x}$-ray probe experiments $[4,5]$ as well as sequential diffractive imaging [6] on a femtosecond to picosecond time scale. Further, direct measurements of the temporal coherence properties will be possible by making use of a linear autocorrelation $[7,8]$. The set-up is based on geometric wavefront beam splitting, which has successfully been implemented at an autocorrelator at FLASH [9]. The x-ray FEL pulses are split by a sharp edge of a silicon mirror coated with multilayers. Both partial beams will then pass variable delay lines. For different photon energies the angle of incidence onto the multilayer mirrors will be adjusted in order to match the Bragg condition. For a photon energy of $\mathrm{h} v=20 \mathrm{keV}$ a grazing angle of $\theta=0.57^{\circ}$ has to be set, which results in a footprint of the beam $(6 \sigma)$ on the mirror of $\mathrm{l}=98 \mathrm{~mm}$. At this photon energy the reflectance of a Mo/ $\mathrm{B}_{4} \mathrm{C}$ multi layer coating with a multilayer period of $\mathrm{d}=3.2 \mathrm{~nm}$ and $\mathrm{N}=200$ layers amounts to $\mathrm{R}=0.92$. In order to enhance the maximum transmission for photon energies of $h v=8$ $\mathrm{keV}$ and below, a Ni/ $\mathrm{B}_{4} \mathrm{C}$ multilayer coating can be applied beside the $\mathrm{Mo} / \mathrm{B}_{4} \mathrm{C}$ coating for this spectral region. Because of the different incidence angles, the path lengths of the beams will differ as a function of wavelength. Hence, maximum delays between $+/-2.5 \mathrm{ps}$ at $\mathrm{h} v=20 \mathrm{keV}$ and up to $+/-23 \mathrm{ps}$ at $\mathrm{h} v=5 \mathrm{keV}$ will be possible.
\end{abstract}

\section{INTRODUCTION}

In this paper a novel x-ray split-and-delay unit based on wavefront beam splitting and multilayer mirror coatings is described. It covers photon energies between $h v=5 \mathrm{keV}$ and $\mathrm{h} v=20 \mathrm{keV}$. With this spectral range the SDU will enable jitter-free pump-probe experiments at the HED experiment [1] at the SASE 2 undulator of the European XFEL [2]. Due to the high absorbance and the small reflectivity at large incident angles a grazing incident geometry is utilized. For the XUV- and soft x-ray spectral regime such a set-up has successfully been integrated into the FLASH SASE FEL [9]. With this device the spatio-temporal coherence properties [7,8] as well as the pulse duration [10] of a soft X-ray FEL have successfully been measured for the first time. Further, ionization dynamics in expanding clusters have been investigated by XUV pump / XUV probe spectroscopy [4], the ultrafast heating of hydrogen has been studied [5] and femtosecond sequential imaging has been realized for the first time [6]. 
The new SDU at the European XFEL will enable similar experiments in the x-ray spectral regime. While for the energy range of FLASH carbon coated silicon mirrors still yield a sufficient reflectivity at photon energies up to $\mathrm{h} v=200 \mathrm{eV}$, this will not be the case for the hard x-ray pulses of the European XFEL. Therefore, Si-substrates coated with multilayers will be utilized.

In order to evaluate the capability of the device to measure the temporal coherence of the hard X-ray pulses timedependent wave-optics simulations have been performed by means of Synchrotron Radiation Workshop (SRW) software for x-ray pulses at hv $=15 \mathrm{keV}$. Main features of the optical layout, including diffraction on the beam splitter edge and optics imperfections measured with a NOM slope measuring profiler are taken into account. The impact of these effects on the characterization of the temporal properties of XFEL pulses are analyzed. An approach based on a Fourier Transformation (FT) allows for the evaluation of the temporal coherence despite of large wavefront distortions caused by the optics imperfections.

\section{OPTICAL CONCEPT}

The high absorbance and the small reflectivity at large incident angles are severe limitations for optical instrumentation in the $\mathrm{x}$-ray range and therefore demand for a grazing incident geometry. The optical concept meets various requirements, like: A high reflectivity, transmission of the whole spatial beam-profile, delay with sub-fs resolution, a large delay range, and the wide photon energy range of the XFEL $(5-20 \mathrm{keV})$. These properties have to be achieved with a minimal disturbance of the beam position and direction, a high mechanical stability making a temporal resolution in the sub 100 attosecond regime feasible. The design incorporates elements, which allow a realization of the SDU in a practicable size.

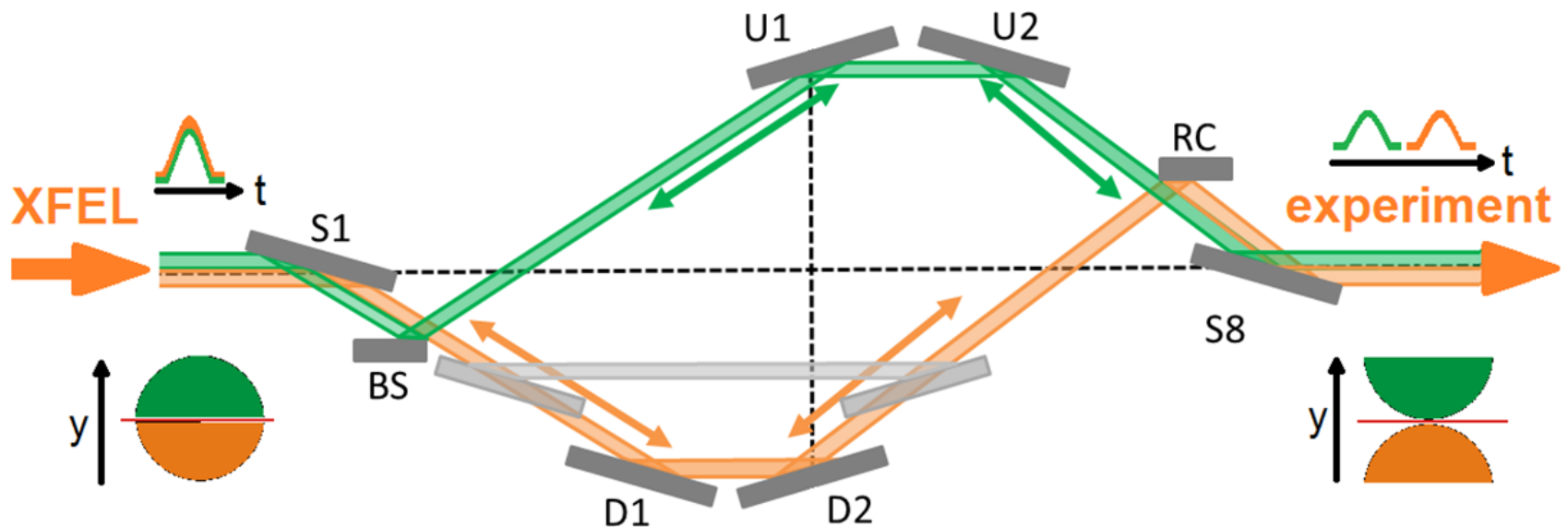

Fig.1: Schematic drawing of the optical layout of the x-ray split- and delay-unit.

In order to meet these requirements a point symmetric optical concept based on a geometrical wavefront beam splitter and multilayer Bragg coatings which permit larger grazing angles has been developed. The whole set-up of the optical pathway is schematically shown in Fig. 1. The XFEL beam enters the SDU from the left side and is reflected by the first mirror (S1) downwards in the direction of the beam splitter (BS). The lower green part of the beam is reflected into the upper delay arm while the upper orange part passes the sharp edge in the direction of the lower delay arm. The mirrors of both delay arms can be moved along the split beam direction in order to introduce a temporal delay between both partial beams. After the orange beam has passed the lower delay line it is reflected by the recombination mirror (RC) in the direction of the last mirror (S8). The green beam passes the sharp edge of the recombination mirror unaffected. Thus, in this point symmetric concept the recombination mirror acts as the counterpart of the beam splitter. The last mirror (S8) reflects both beams into their original direction. It should be noted that the beam shape of both arms is rotated by $180^{\circ}$ due to the odd number of reflections. In order to perform experiments the beams have to be overlapped. This can be achieved by slightly rotating the recombination mirror, RC.

As already mentioned the multilayer mirrors are intended to work at Bragg angles depending on the photon energy. For photon energies from $\mathrm{h} v=5 \mathrm{keV}$ to $\mathrm{h} v=20 \mathrm{keV}$ multilayers will be used on the mirrors which possess high reflectivity. 


\section{MECHANICAL LAYOUT}

The projected sub-fs resolution as well as the essential pointing stability of the partial beams demand an extensive mechanical stability of the $6 \mathrm{~m}$ long construction. A picture of the SDU during the construction phase is shown in figure 2. An intrinsic mechanical stabilization of the entire system is achieved by increasing the stiffness of the whole system. Thereby vibrations are significantly reduced. To ensure the mechanical sturdyness all components are mounted inside an optical bench which consists of an octagonal structure of stainless steel. As discussed before the FEL beam is divided geometrically and both partial beams travel along two paths whose lengths can be adjusted. The path-length difference of one beam with respect to the other and in consequence the temporal delay is changed by moving the mirrors of both arms along guide rails. These rails are supported by stainless steel frames. The mirrors are mounted to girders that are moved along the guide rails by means of recirculating ball drives.

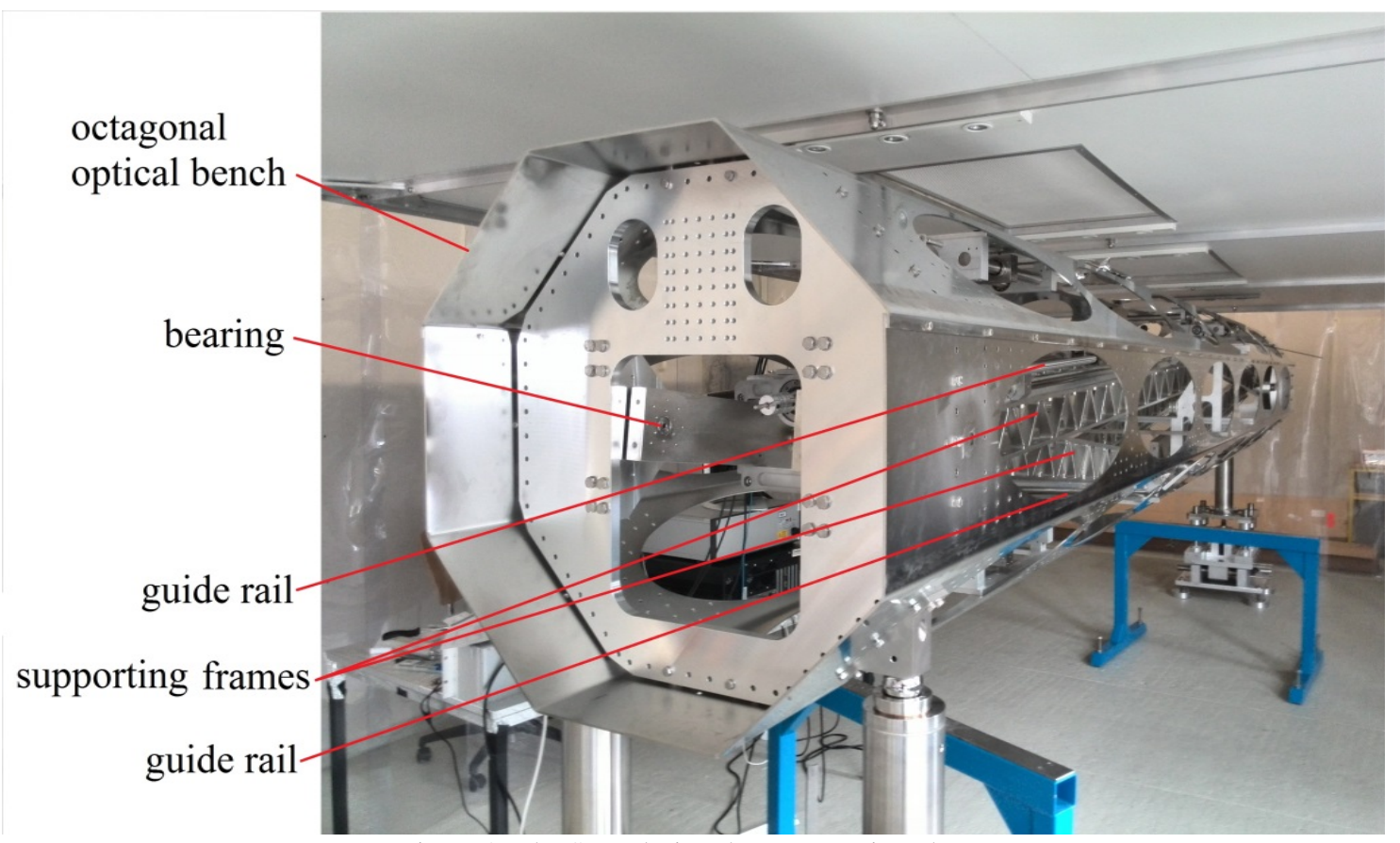

Figure 2: The SDU during the construction phase.

In order to adjust the correct angles for different photon energies all mirrors are turnable and the angle of the guide rails is variable. By moving the mirror the path length which the beam travels along the hypotenuse instead of the (shorter) adjacent of a triangle is varyied, compare Fig. 1. To obtain the designed sub-100 attosecond resolution of the delay this longitudinal motion of the mirrors has to be very precise. Under an grazing angle of $\theta=0.57^{\circ}$ (for hv $=20 \mathrm{keV}$ ) a movement of the mirror of $\Delta \mathrm{l}=10 \mu \mathrm{m}$ results in a pathlength difference of the light of $1.9 \mathrm{~nm}$ which corresponds to a temporal delay of $\Delta \mathrm{t}=6$ as. If the grazing angle is $\theta=2.28^{\circ}$ (for $\mathrm{h} v=5 \mathrm{keV}$ ) the corresponding delay for a movement of $\Delta \mathrm{l}=10 \mu \mathrm{m}$ is $\Delta \mathrm{t}=100$ as. Since the grazing angles of multilayer mirrors depend on the photon energy, an adjustability of the angles of incidence is required. The mirror mountings will therefore posses an angular precission of better than $\Delta \alpha=1 \mu \mathrm{rad}$. For the last mirrors, that reflect the beam to the experiment an additional piezo driven fine tuning is foreseen. In order to provide the experiments with an unaffected XFEL beam the whole optical bench can be moved horizontally so that the beam does not hit any mirror of the SDU. 


\section{MIRRORS}

\subsection{Multilayer coating}

For photon energies between $5 \mathrm{keV}<\mathrm{h} v<10 \mathrm{keV} \mathrm{Ni} / \mathrm{B}_{4} \mathrm{C}$ coatings with a period of $\mathrm{d}=4 \mathrm{~nm}$ will be applied on all mirrors except for BS and RC which reflect the beam at Bragg angles twice as large as the others. Here W/ $\mathrm{B}_{4} \mathrm{C}$ coatings with a period of $d=1.96 \mathrm{~nm}$ are applied since $\mathrm{Ni} / \mathrm{B}_{4} \mathrm{C}$ coatings with a period below $\mathrm{d}=3 \mathrm{~nm}$ cannot be manufactured due to interdiffusion processes between the layers. For photon energies above hv $=10 \mathrm{keV} \mathrm{Mo/B}{ }_{4} \mathrm{C}$ multilayers with a period of $\mathrm{d}=3.2 \mathrm{~nm}$ are used. Since for multilayers the grazing angle depends on the wavelength, the mirrors have to be aligned for different wavelengths. Hence, the maximum possible delay will vary as a function of the photon energy between $\Delta \mathrm{t}_{\max }=23 \mathrm{ps}$ at hv $=5 \mathrm{keV}$ and $\Delta \mathrm{t}_{\max }=2.5 \mathrm{ps}$ at hv $=20 \mathrm{keV}$, see table 1 .

It is possible to choose between different mirror coatings by moving the whole set-up horizontally. For two-color experiments with fundamental and third harmonic radiation a third coating has to be applied on the mirrors S1 and S8 because these two mirrors will have to reflect both photon energies at the same Bragg angle. The footprint of the beam on the mirrors depends on the incidence angle and on the photon energy. It varies between l $=84 \mathrm{~mm}$ at hv $=5 \mathrm{keV}$ and $\mathrm{l}=98 \mathrm{~mm}$ at $\mathrm{hv}=20 \mathrm{keV}$ for S1, S8 and the mirrors in the delay lines U1/U2 and D1/D2. Since the Bragg-angle is twice as large for BS and RC the footprint will be about two times smaller here.

Table 1: The total transmission and the maximum delay times

\begin{tabular}{|c|c|c|c|c|c|c|}
\hline $\begin{array}{c}\text { Photon energy } \\
{[\mathrm{keV}]} \\
\end{array}$ & $\begin{array}{c}\text { Angle BS/RC } \\
{\left[^{\circ}\right]} \\
\end{array}$ & $\begin{array}{c}\text { Multilayer } \\
\text { Material }\end{array}$ & $\begin{array}{c}\text { Angle S1/S8, } \\
\left.\text { U1/U2, D1/D2 [ }{ }^{\circ}\right]\end{array}$ & $\begin{array}{c}\text { Multilayer } \\
\text { Material }\end{array}$ & $\begin{array}{c}\text { Delay } \\
\text { [ps] }\end{array}$ & Transmission \\
\hline 4 & 4,58 & $\mathrm{Ni} / \mathrm{B} 4 \mathrm{C}$ & 2,29 & W/B4C & 36 & 0,16 \\
\hline 5 & 3,66 & $\mathrm{Ni} / \mathrm{B} 4 \mathrm{C}$ & 1,83 & W/B4C & 23 & 0,23 \\
\hline 6 & 3,07 & $\mathrm{Ni} / \mathrm{B} 4 \mathrm{C}$ & 1,54 & W/B4C & 16 & 0,33 \\
\hline 8 & 2,30 & $\mathrm{Ni} / \mathrm{B} 4 \mathrm{C}$ & 1,15 & W/B4C & 9 & 0,47 \\
\hline 10 & 1,83 & $\mathrm{Ni} / \mathrm{B} 4 \mathrm{C}$ & 0,92 & W/B4C & 6 & 0,21 \\
\hline 12 & 1,54 & $\mathrm{Ni} / \mathrm{B} 4 \mathrm{C}$ & 0,77 & W/B4C & 4 & 0.08 \\
\hline 8 & 2,86 & Mo/B4C & 1,43 & Mo/B4C & 14 & 0.09 \\
\hline 10 & 2,28 & Mo/B4C & 1,14 & Mo/B4C & 9 & 0,29 \\
\hline 12 & 1,90 & Mo/B4C & 0,95 & Mo/B4C & 6 & 0,35 \\
\hline 15 & 1,52 & Mo/B4C & 0,76 & Mo/B4C & 4 & 0,41 \\
\hline 18 & 1,27 & $\mathrm{Mo} / \mathrm{B} 4 \mathrm{C}$ & 0,64 & $\mathrm{Mo} / \mathrm{B} 4 \mathrm{C}$ & 3 & 0,43 \\
\hline 20 & 1,14 & Mo/B4C & 0,57 & Mo/B4C & 2.5 & 0,23 \\
\hline
\end{tabular}

Since this is not possible with a single coating, a novel two-color multilayer Bragg mirror has been developed [12]. This mirror consists of a Si substrate that is coated with two different types of multilayer systems, $\mathrm{n}=120 \mathrm{Mo} / \mathrm{B}_{4} \mathrm{C}$ layers with a periodicity of $d=3.2 \mathrm{~nm}$ directly on the substrate and $\mathrm{n}=4 \mathrm{Ni} / \mathrm{B}_{4} \mathrm{C}$ layers with a periodicity of $\mathrm{d}=11.85 \mathrm{~nm}$ on top. Fundamental radiation with photon energies between $3 \mathrm{keV}$ and $9 \mathrm{keV}$ is reflected by a $\mathrm{Ni} / \mathrm{B}_{4} \mathrm{C}$ multilayer system while the third harmonic ( $9 \mathrm{keV}<\mathrm{hv}<20 \mathrm{keV}$ ) passes this system and is reflected by the $\mathrm{Mo} / \mathrm{B}_{4} \mathrm{C}$ multilayers. The reflectance of this novel two-color hard $\mathrm{x}$-ray multilayer Bragg mirror has experimentally been evaluated at the ESRF. The reflectance is $\mathrm{R}=0.73$ and $\mathrm{R}=0.78$ for the fundamental photon energies at $\mathrm{hv}=5 \mathrm{keV}$ and $\mathrm{hv}=6 \mathrm{keV}$, respectively. For the corresponding third harmonic radiation reflectances of $\mathrm{R}=0.70$ and $\mathrm{R}=0.75$ at hv $=15 \mathrm{keV}$ and $\mathrm{h} v=18 \mathrm{keV}$ were determined.

\subsection{Mirror substrate}

For the simulations the knowledge of the mirror profiles is required in order to evaluate the impact of figure deviations on the wave front of the XFEL pulse. For this purpose the mirrors were inspected by use of the Nanometer Optic component measuring Machine (NOM) [11] at the BESSY-II metrology laboratory at HZB in Berlin. The NOM allows 
a precise measurement of such optics by use of slope measuring deflectometry with sub-nm precision [12] and a spatial resolution of $1.7 \mathrm{~mm}$ up to a length of $1200 \mathrm{~mm}$ [13]. Figure 3 shows the mirror profile that was used for the wavefront propagation.

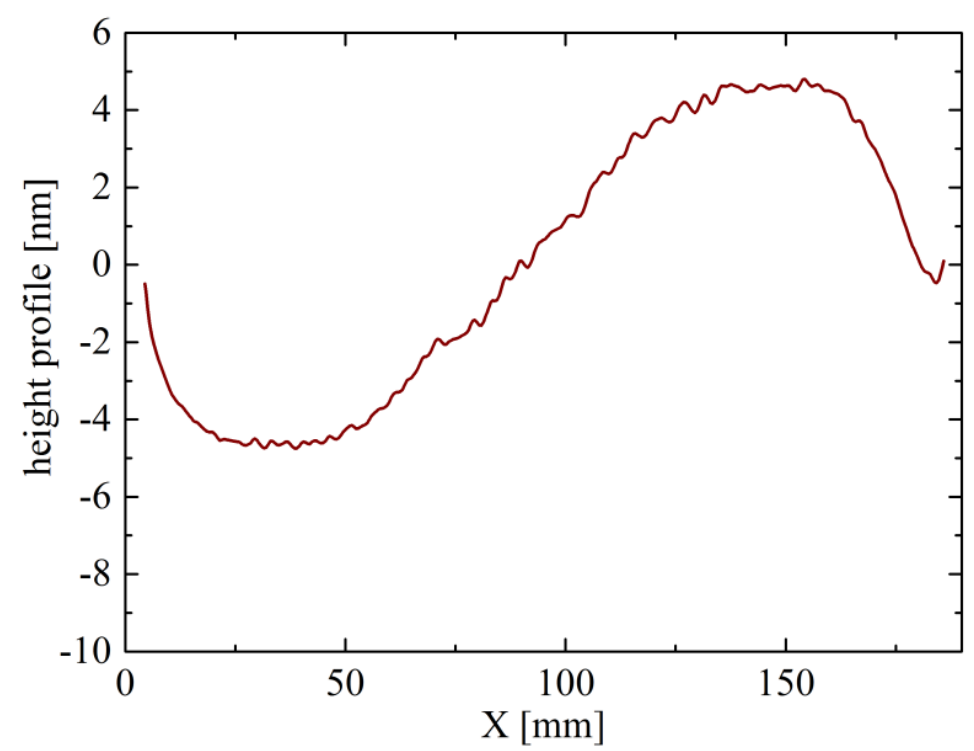

Figure 3: The height profile of the mirror used for the wavefront propagation simulation.

The $l=190 \mathrm{~mm}$ long mirror shows a sine like surface profile with a peak-to-valley error of $\Delta \mathrm{h}=9.5 \mathrm{~nm}$. This profile was used to simulate the reflection of the beam at BS and RC.

\subsection{Wavefront propagation}

The SRW software based on a Fourier optics approach and WPG framework provides a powerful tool for the simulating the wavefront propagation of XFEL pulses [14, 15]. It offers a comprehensive and extendable toolbox that is capable of solving a wide range of XFEL optics problems.

The software takes into account the optics imperfections and allows modelling essential optical elements such as grazing incidence plane and focussing mirrors and gratings. The optical performance of the SDU mirrors was evaluated by means of a Python script that incorporates SRW and WPG library.

The wavefront propagation simulations were carried out for photon energies of hv $=5 \mathrm{keV}$ and $15 \mathrm{keV}$ for Gaussian pulses with a coherence time that amounts to $t_{c}=0.243$ fs. The angular divergence of the pulses was $\Theta_{5}=3.87$ mrad and $\Theta_{15}=1.8 \mathrm{mrad}$ for $\mathrm{hv}=5 \mathrm{keV}$ and $15 \mathrm{keV}$, respectively. After leaving the undulator the beam propagates towards the SDU which will be located $\mathrm{z}=846 \mathrm{~m}$ behind the undulator. At BS the beam is split into two partial beams that are delayed with respect to each other. Then the two partial beams propagate $\mathrm{z}=130 \mathrm{~m}$ and irradiate the detector in the experimental area. Figure 4 shows the two partial beams on the detector for a photon energy of hv $=5 \mathrm{keV}$ with perfect mirrors in the SDU (a) and for mirrors with the mirror profile shown in figure 3 (b). Within the $\mathrm{l}=40 \mathrm{~mm}$ long area that is irradiated by the footprint of the beam the height error of the mirror amounts to $\Delta \mathrm{h}=4.7 \mathrm{~nm}$. In figure 4 (a) only fringes from diffraction at BS and RC are visible. In figure 4(b) also fringes caused by wavefront distortions occur. Figure 5 shows the corresponding situation at a photon energy of hv $=15 \mathrm{keV}$. 


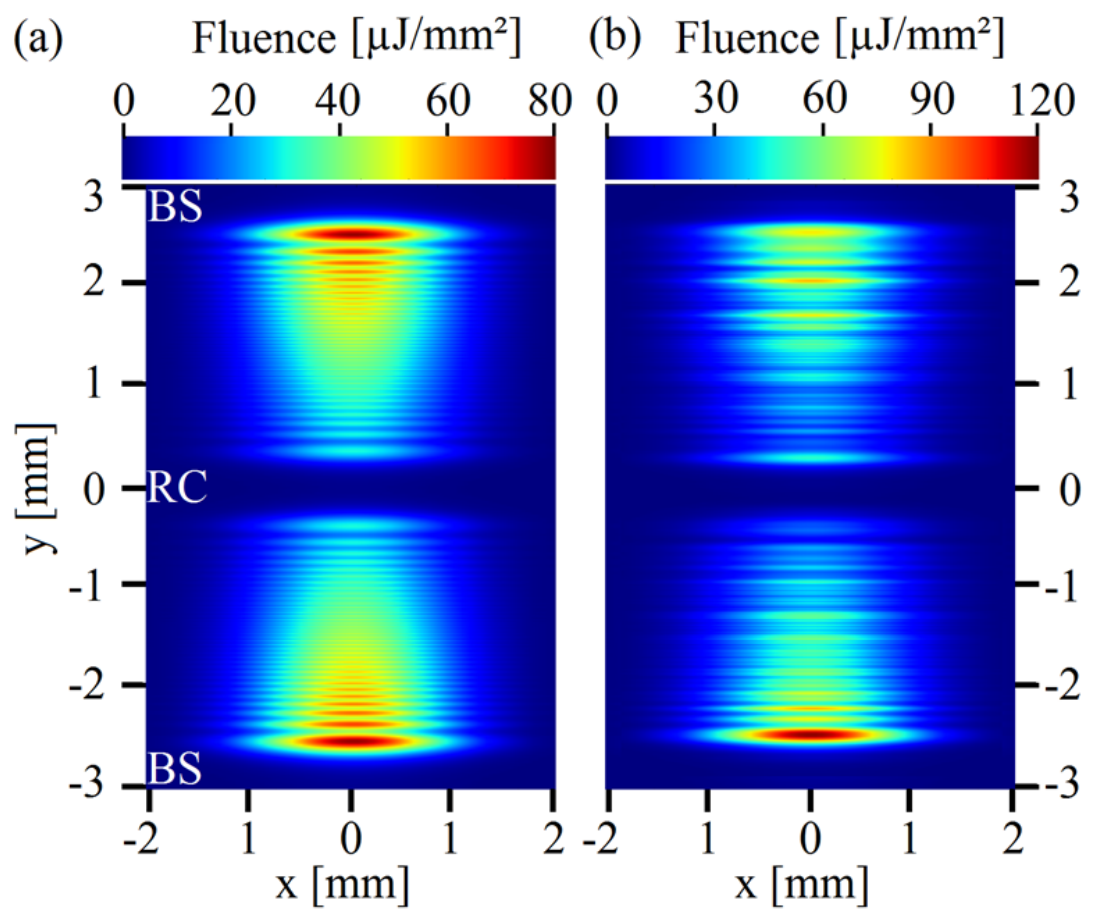

Figure 4: The two partial beams on the detector for a photon energy of hv $=5 \mathrm{keV}$ with perfect mirrors in the SDU (a) and for mirrors with the mirror profile (b).

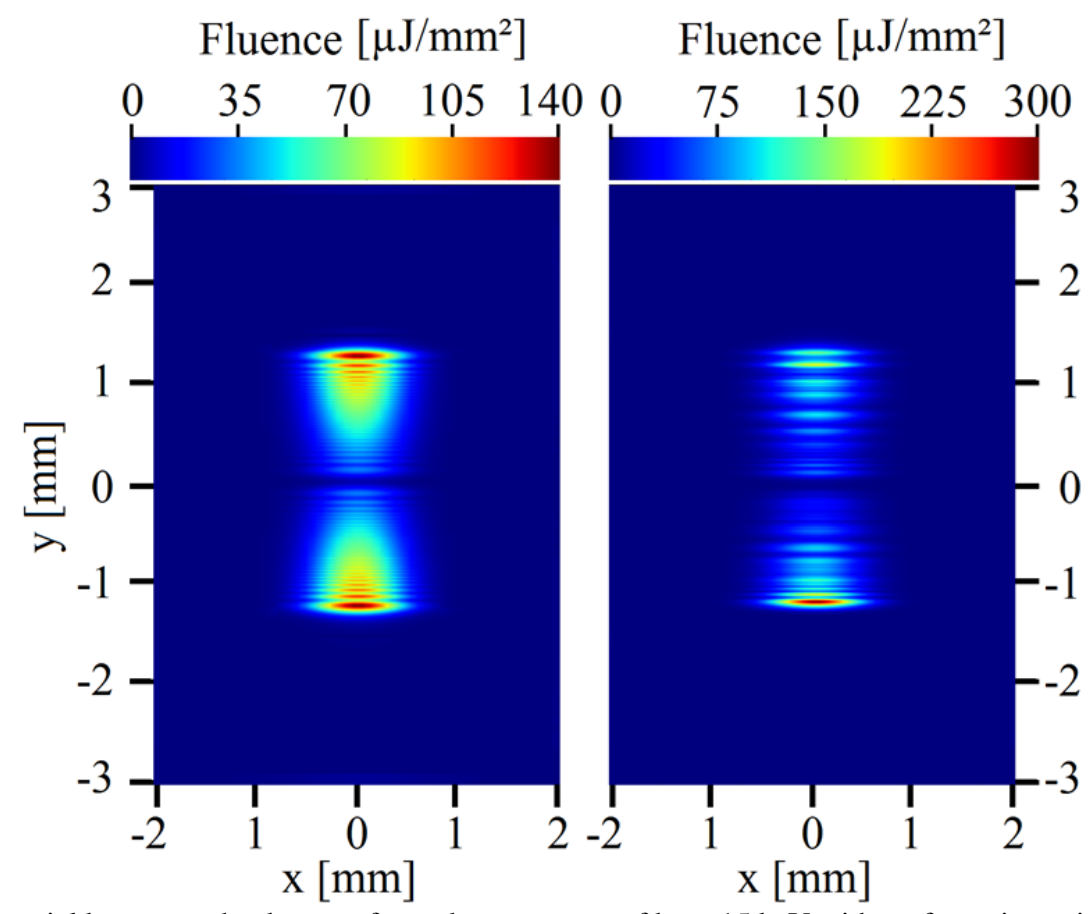

Figure 5: The two partial beams on the detector for a photon energy of hv $=15 \mathrm{keV}$ with perfect mirrors in the SDU (a) and for mirrors with the mirror profile (b). 


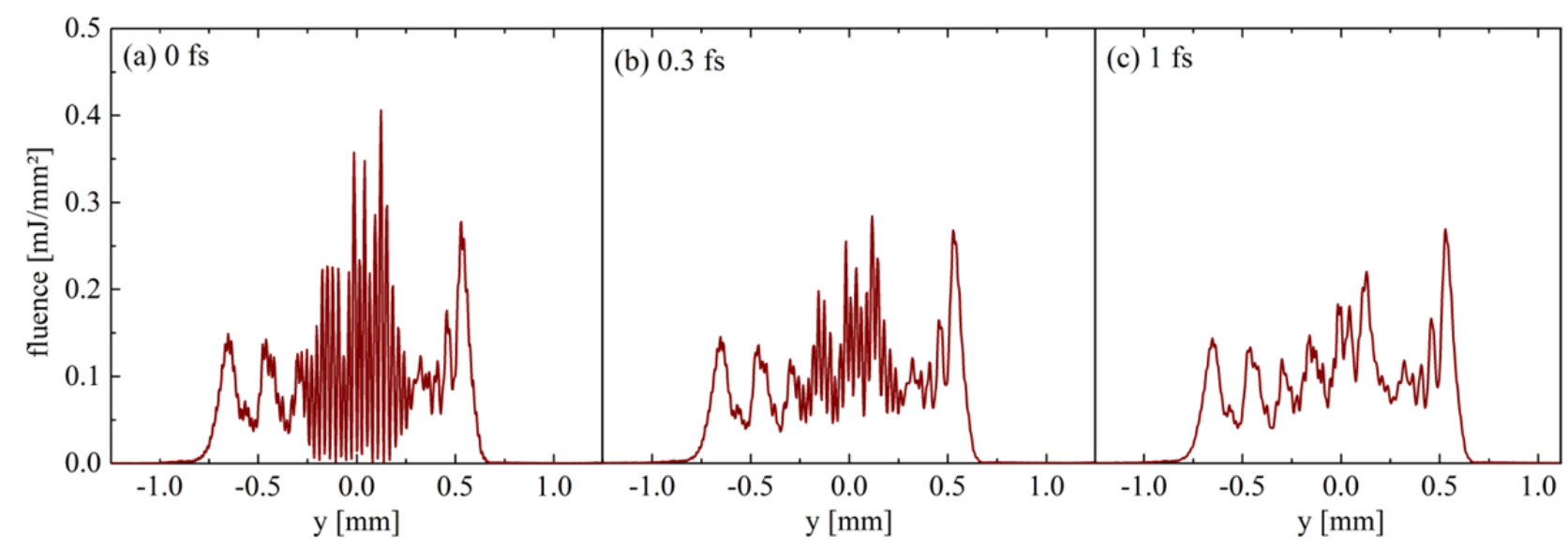

Figure 6: A vertical cut through the interference pattern for three different delays at (a) $\Delta t=0 \mathrm{fs}$, (b) $\Delta \mathrm{t}=0.21 \mathrm{fs}$, (c) $\Delta t=1 \mathrm{fs}$.

A measurement of the temporal coherence is simulated for $\mathrm{h} v=15 \mathrm{keV}$. The vertical position $\mathrm{y}$ of $\mathrm{RC}$ is aligned to accept $d_{y}=0.8 \mathrm{~mm}$ of the partial beam from the lower delay branch (orange in Fig. 1). Thus, also the other partial beam is cut by the edge of RC such that $\mathrm{d}_{\mathrm{y}}=0.8 \mathrm{~mm}$ will pass. Consequently only the first $l=30 \mathrm{~mm}$ of the mirror profile are irradiated. The peak-to-valley height error within this footprint amounts to $\Delta \mathrm{h}=4.7 \mathrm{~nm}$. The two partial beams then propagate $130 \mathrm{~m}$ before they irradiate the detector in the experimental hutch. Figure 6 shows a vertical cut through the interference fringes at a delay of (a) $\Delta \mathrm{t}=0 \mathrm{fs}$, (b) $\Delta \mathrm{t}=0.3 \mathrm{fs}$ and (c) $\Delta \mathrm{t}=1 \mathrm{fs}$. The fringes caused by diffraction at the edges of the BS and RC and by the wavefront distortions are clearly visible in all three cases. For a delay of $\Delta t=0 \mathrm{fs}$ fully modulated fringes from time-dependent two-beam interference appear. The modulation of these fringes decreases for a delay of $\Delta \mathrm{t}=0.3 \mathrm{fs}$ and completely vanishes at $\Delta \mathrm{t}=1 \mathrm{fs}$. The temporal coherence of the XFEL pulses can be determined by means of a Fourier transform [FT] of the interference patterns for different delays. Figure 7 shows the FT for the three delays discussed here.

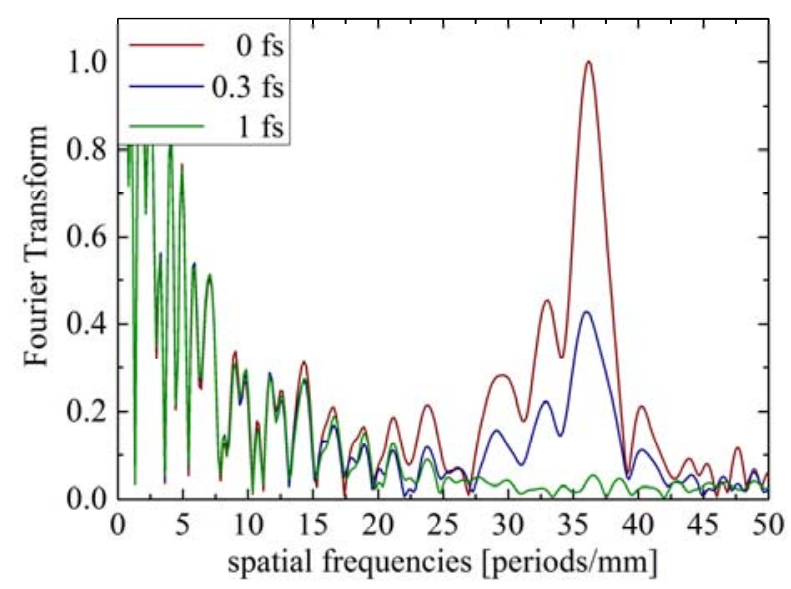

Figure 7: The Fourier transform of the interference patterns shown in Figure 3.

The FT signal from the wavefront disturbances and diffraction at the edges of BS and RC is spread over the entire spatial frequency range with increasing magnitudes at lower frequencies for all delays. The maximum from timedependent two-beam interference appears at a spatial frequency of $f=36$ fringes $/ \mathrm{mm}$. The result of a simulated measurement of the temporal coherence of XFEL pulses is shown in figure 8. 


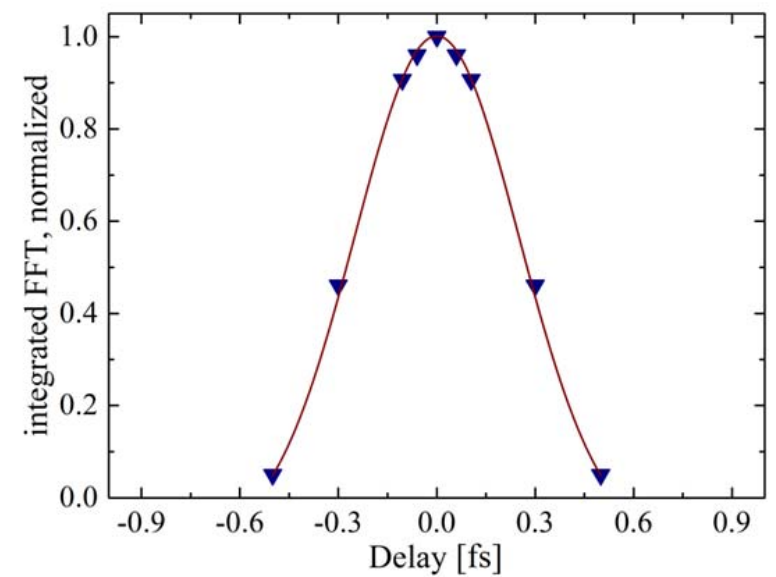

Figure 8: The FT signal from time-dependent two-beam interference plotted versus the delay.

The data can be approximated with a Gaussian function with an r.m.s. width of $\tau_{\mathrm{c}}=0.247 \mathrm{fs}$. In comparison with the coherence time of the Gaussian pulse that was assumed for the simulation the error amounts to only 4 as or $1.6 \%$.

\section{CONCLUSION}

A new split- and delay-unit for the European XFEL is designed and constructed for photon energies between hv $=5 \mathrm{keV}$ and $\mathrm{h} v=20 \mathrm{keV}$. This SDU will serve the users with two time delayed $\mathrm{x}$-ray pulses for x-ray pump / $\mathrm{x}$-ray probe experiments and it will enable a characterization of the temporal properties of the XFEL. Multilayer coated mirrors will be used in order to provide for a maximum total transmission between $56 \%$ at hv $=18 \mathrm{keV}$ and $48 \%$ at $\mathrm{hv}=8 \mathrm{keV}$, respectively and $26 \%$ at hv $=5 \mathrm{keV}$. Wavefront propagation simulations performed with SRW and WPG framework show that the SDU will be well suited for a measurement of the temporal coherence of the XFEL pulses.

\section{ACKNOWLEDGEMENT}

This work is supported by the BMBF (project: 05K10PM2 and 05K13PM1).

\section{REFERENCES}

[1] M. Nakatsumi et al., Scientific Instrument High Energy Density Physics (HED), XFEL.EU TR-2014-001 (2014)

[2] M. Altarelli et al., The European X-Ray Free Electron Laser Technical Design Report, DESY Hamburg (2006)

[3] S. Roling et al., Proc. SPIE 8504, 850407 (2012)

[4] M. Krikunova et al., J. Phys. B: At. Mol. Opt. Phys. 45, 105101 (2012)

[5] U. Zastrau et al., Phys. Rev. Lett. 112, 105002 (2014)

[6] C.M. Günther et al., Nat. Phot 5, 99-102 (2011).

[7] R. Mitzner et al., Opt. Expr. 16, 19909-19919 (2008)

[8] S. Roling et al., Phys. Rev. ST Accel. Beams 14, 080701 (2011) 
[9] M. Wöstmann et al., J. Phys. B: At. Mol. Opt. Phys. 46, 164005 (2013)

[10] R. Mitzner et al., Phys. Rev. A 80, 025402 (2009)

[11] F. Siewert et al., Nucl. Instr. Meth. in Phys. Res. A 635, 52 (2011)

[12] F. Siewert et al., Opt. Expr. 20, 4526 (2012)

[13] F. Siewert et al., Nucl. Instr. Meth. in Phys. Res. A 710, 422 (2013)

[14] O. Chubar et al., Nucl. Instr. Meth. in Phys. Res. A 593, 30 (2008)

[15] A. Buzmakov et al., Proc. SPIE 9209, 9209-32 (2014) 